\title{
Anti-apoptotic effect of claudin-1 in tamoxifen- treated human breast cancer MCF-7 cells
}

\author{
Harue Akasaka ${ }^{1}$, Fuyuki Sato ${ }^{1 *}$, Satoko Morohashi', Yunyan Wu ${ }^{1,2}$, Yang Liu ${ }^{1,2}$, Jun Kondo ${ }^{1}$, Hiroki Odagiri ${ }^{3}$, \\ Kenichi Hakamada ${ }^{3}$, Hiroshi Kijima ${ }^{1}$
}

\begin{abstract}
Background: Claudin-1 is a membrane protein of tight junctions, and is associated with the development of various cancers. However, the significance of claudin-1 expression in cancer cells is not well understood. Here, we showed for the first time the anti-apoptotic effect of claudin-1 in human breast cancer MCF-7 cells.

Methods: Human breast cancer MCF-7 and T47 D cells were treated with or without tamoxifen, siRNA against claudin-1, or tamoxifen and claudin-1 siRNA. The samples were analyzed by RT-PCR, Western blotting or immunofluorescent staining.

Results: The expression of claudin-1 was upregulated in tamoxifen-treated MCF-7 cells, whereas the expression of claudin-1 was not altered in tamoxifen-treated T47 D cells. Knockdown of claudin-1 by siRNA increased the amount of poly (ADP-ribose) polymerase (PARP) regardless of tamoxifen treatment in MCF-7 cells, but not T47 D cells. In the cell membranes of the MCF-7 cells, tamoxifen treatment increased the amount of claudin-1, but decreased the amount of $\beta$-catenin. Claudin-1 siRNA increased the amount of E-cadherin in the cytoplasm of the MCF-7 cells as well as the amount of $\beta$-catenin in their cell membranes.
\end{abstract}

Conclusion: These results indicate that claudin-1 has anti-apoptotic effects, and is involved in the regulation of the expression and subcellular localization of $\beta$-catenin and E-cadherin in MCF-7, but not T47 D cells.

\section{Background}

Breast cancer is the second most common cause of female mortality in United States. The breast cancer incidence and mortality rates were about 190,000 and 40,000, respectively, in 2009 [1]. The majority of breast cancers are sporadic, and most risk factors for the disease are related to estrogen exposure. This suggests that insufficient apoptosis in cancer cells is involved in their survival as insuffcient apoptosis leads to the development of chemotherapy resistance and carcinogenesis [2].

Tamoxifen is one of most widely used anti-estrogen drugs for the treatment of human breast cancer [3]. Tamoxifen treatment leads to a rapid decrease in number of S-phase cells, an accumulation of cells in the G1fraction [4], and the induction of apoptosis in vivo and vitro [5-7]. Tamoxifen induces apoptosis through several distinct pathways including a mitochondria-dependent

\footnotetext{
* Correspondence: fsato@cc.hirosaki-u.ac.jp

'Department of Pathology and Bioscience, Hirosaki University Graduate

School of Medicine, Hirosaki 036-8562, Japan

Full list of author information is available at the end of the article
}

pathway, the induction of c-Myc, the activation of members of the mitogen-activated protein kinases (MAPK) family, and the upregulation of p53 [7-11]. However, the detailed molecular mechanisms by which tamoxifen induces apoptosis are not well understood.

Tight junctions and adherens junctions proteins, including claudins, E-cadherin, $\beta$-catenin, and ZOs proteins, are responsible for the maintenance of epithelial cell-cell adhesion and defining cell polarity, and are also involved in cell signaling events [12]. Changes in claudin expression are also involved in invasion, metastasis, and colony formation in various cancer cells [13-15]. In a previous study, the mRNA expression of claudin-1 was decreased in the tumor group compared with the control (normal) group in breast cancer tissues [16]. Decreased expression of claudin-1 was also correlated with breast cancer recurrence [17]. However, the relationship between claudin-1 and chemotherapy is poorly understood.

In the present study, we investigated the relationship between claudin-1 and tamoxifen treatment in human 
breast cancer MCF-7 and T47 D cells. The expression of claudin-1 was upregulated by tamoxifen treatment in MCF-7 cells. Combination treatment with both claudin1 siRNA and tamoxifen significantly increased the amount of cleaved PARP. Knockdown of claudin-1 affected the expression and subcellular localization of $\beta$ catenin and E-cadherin in MCF-7 cells. Our results suggest that claudin-1 has an anti-apoptotic effect, involving the regulation of $\beta$-catenin and E-cadherin, in MCF-7 cells.

\section{Methods}

\section{Cell culture and treatment}

MCF-7 and T47 D cells were obtained from the American Type Culture Collection (ATCC, Manassas, VA, USA). These cells were cultured in Dulbecco's Modified Eagle's Medium-high glucose (Sigma Chemical Co., St. Louis, MO, USA) supplemented with $10 \%$ fetal bovine serum at $37^{\circ} \mathrm{C}$ in a humidified atmosphere of $95 \%$ air and $5 \% \mathrm{CO}_{2}$. When the MCF-7 cells were treated with $40 \mu \mathrm{M}$ of tamoxifen (Sigma) for $20 \mathrm{~h}$, apoptotic reactions were detected as described below. However, the incubation with $40 \mu \mathrm{M}$ of tamoxifen for more than $24 \mathrm{~h}$ resulted in the severe toxicity to cells, and more than $90 \%$ of cells were detached from the plates (data not shown). Therefore, we treated the cells with $40 \mu \mathrm{M}$ of tamoxifen for $20 \mathrm{~h}$ in the follow experiments. In addition, we treated MCF-7 cells with 1,10 or $20 \mu \mathrm{M}$ of tamoxifen for $48 \mathrm{~h}$ in some experiments to observe the longer effects.

\section{Reverse transcription-polymerase chain reaction (RT-PCR) and real-time PCR}

Total RNA was isolated using an RNeasy RNA isolation kit (QIAGEN, Hilden, Germany). First-strand cDNA was synthesized from $1 \mu \mathrm{g}$ of total RNA using ReverTra Ace (TOYOBO, Osaka, Japan). RT-PCR was performed using an aliquot of first-strand cDNA as a template under standard conditions with Taq DNA polymerase (QIAGEN). The primers were designed to perform optimal RT-PCR by DNASIS software, and the primers used were as follows: claudin-1-F: 5'-CAGCTGTTGGGCTTCATTCTC3', claudin-1-R: 5'-ATCACTCCCAGGAGGATGCC-3'; claudin 4-F: 5'-ATGGCCTCCATGGGGCTACA-3', claudin 4-R: 5'-AGCGAGTCGTACACCTTGCA-3'; E-cadherin-F: 5'-ACATTGTCACCTCGCAGAC-3', E-cadherinR: 5'-GCGGATTGTAGAAGTCTTGG-3'; GAPDH-F: 5'CCACCCATGGCAAATTCCATGGCA-3', GAPDH-R: 5'AGACCACCTGGTGCTCAGTGTAGC-3'. The amplified products of claudin-1, claudin-4, E-cadherin, and GAPDH were $277 \mathrm{bp}, 208 \mathrm{bp}, 336 \mathrm{bp}$, and $696 \mathrm{bp}$, in length, respectively. The cDNA for claudin-1, claudin-4, E-cadherin, and GAPDH were amplified for up to 25 cycles.
The PCR products were separated on $1.5 \%(\mathrm{w} / \mathrm{v})$ agarose gels.

The real-time PCR was carried out using SYBER Green Master Mix (Applied Biosystems, Tokyo, Japan). The primers used as follows: claudin-1-F: 5'-AGATGAGGATGGCTGTCATTGG -3', claudin-1-R: 5'-CATGCT GTGGCAGCTAAAATAGC-3'; E-cadherin-F: 5'-ACAT TGTCACCTCGCAGAC-3', E-cadherin-R: 5'-GCGGAT TGTAGAAGTCTTGG-3'; 18 S rRNA-F: 5'-GTAACC CGTTGAACCCCATT-3', 18 S rRNA-R: 5'-CCATCCAATCGGTAGTAGCG-3'. The amplified products of claudin-1, E-cadherin, and $18 \mathrm{~S}$ rRNA were $72 \mathrm{bp}, 336 \mathrm{bp}$, and $150 \mathrm{bp}$, in length, respectively.

\section{Short interference RNA (siRNA)}

Short interference RNA (siRNA) against claudin-1 were synthesized by QIAGEN. The sequences for the sense and anti-sense claudin-1 siRNA were 5'-r (GCAUGGUAUGGCAAUAGAA) d (TT) -3 ' and 5'-r (UUCUAUUGCCAUACCAUGC) d (TG) -3', respectively. We also used another siRNA against claudin-1 (claudin-1 siRNA2). The sequences for the sense and anti-sense claudin-1 siRNA2 were 5'-r (CGAAAUUGUUACAAUAGAA) d (TT)-3' and 5'-r (UUCUAUUGUAACAAUUUCG) d (TT)-3'. The negative control (scrambled) siRNA sequences were 5'-r (UUCUCCGAACGUGUCACGU) d (TT)-3' and 5'-r (ACGUGACACGUUCGGAGAA) d (TT)-3'. For the siRNA transfection experiments, MCF-7 and T47 D cells were seeded at $5 \times 10^{4}$ cells per $35-\mathrm{mm}$ well. Twenty-four $\mathrm{h}$ later, the siRNA were transfected into the cells using the Lipofectamine RNA iMAX reagent (Invitrogen, Carlsbad, CA, USA). After transfection, the cells were incubated for $48 \mathrm{~h}$ and subjected to various analyses.

\section{Western Blotting}

The cells transfected with siRNA were lysed using MPER lysis buffer (PIERCE, Rockford, IL, USA). Protein concentrations were determined using the bicinchoninic acid (BCA) assay. The obtained lysates (10 $\mu$ g protein) were subjected to SDS-PAGE, and the acquired proteins were transferred to PVDF membranes (Immobilion P, Millipore, Tokyo, Japan). The membranes were then incubated with antibodies specific for claudin-1 $(1: 10,000)$, claudin-4 (1:20,000), and claudin-7 (1:2,000), which were purchased from Invitrogen; E-cadherin $(1: 1,000)$, which was purchased from Takara, Shiga, Japan; $\beta$-catenin (1:30,000), Bcl-2 $(1: 2,000)$, and p21 $(1: 1,000)$, which were purchased from EPITOMICS, CA, USA; cyclin D1 (1:1,000), which was purchased from Merck, Darmstadt, Germany; PARP $(1: 1,000)$ and cleaved caspase-8 $(1: 10,000)$, which were purchased from Cell Signaling Technology, Inc Danvers, MA, USA; Bax (1:1,000), which was purchased from Santa Cruz, 
CA, USA; p53 (1:2,000), which was purchased from Abcam, Cambridge, UK; and actin $(1: 30,000)$ (Sigma), followed by treatment with horseradish peroxidase-conjugated secondary antibody (IBL, Gunma, Japan). Can Get Signal Immunoreaction Enhancer Solution 1 (TOYOBO) was used to dilute the primary antibody. The ECL, ECL-plus, or ECL-advance Western Blotting Detection System (Amersham, Uppsala, Sweden) was used for detection. The intensity of the bands was quantified by using the National Institute of Health Image computer program. The signal intensities were compensated by actin as internal controls.

\section{Immunofluorescent staining}

MCF-7 and T47 D cells were seeded on a 4-chamber slide glass and incubated overnight. The cells were then washed with phosphate-buffered saline (PBS) and fixed with ice-chilled methanol for $30 \mathrm{~min}$, before being permeabilized with $0.2 \%$ Triton-X-100 in PBS for $30 \mathrm{~min}$. The permeabilized cells were then washed in PBS twice and treated with 5\% normal horse serum in PBS for 30 min (to minimize the non-specific adsorption of antibodies), before being incubated with anti-claudin-1 (1:200), anti- $\beta$-catenin (1:300), or anti-E-cadherin (1:300) antibodies at $4^{\circ} \mathrm{C}$ overnight. The cells were then incubated for $1 \mathrm{~h}$ with goat anti-rabbit IgG antibody conjugated to Alexa 488 dye (Molecular Probes, Inc, Tokyo, Japan), while nuclear staining was performed using 4', 6-diamidino-2-phenylindole (DAPI) or Hoechst 33258. Hoechst 33258 staining was used to examine nuclear condensation. The cells were visualized using confocal laser scanning microscopy (Zeiss, LSM 710, Wetzlar, Germany), and the number of cells that were intensely stained with Hoechst 33258 was counted.

\section{Results}

Tamoxifen treatment induces apoptosis and upregulates the expression of claudin-1 in MCF-7 cells

We investigated the endogenous expression of claudin-1 in two breast cancer cell lines by Western blotting. The endogenous protein expression of claudin-1 was weak in MCF-7 cells, whereas it was abundantly expressed in T47 D cells (Figure 1A and 1B). In addition, E-cadherin was abundantly expressed in both MCF-7 and T47 D cells. Next, we examined the relationship between tamoxifen and claudin-1. We examined how the protein expression of claudin-1 was affected by tamoxifen treatment. MCF-7 and T47 D cells were treated with various concentrations of tamoxifen for $20 \mathrm{~h}$. Cell lysates were prepared from cells and subjected to Western blot analysis. The protein expression of claudin-1 was slightly increased by treatment with $20 \mu \mathrm{M}$ tamoxifen in MCF-7 cells, and the cells treated with 30 or $40 \mu \mathrm{M}$ of tamoxifen treatment showed clear increases in their claudin-1 protein levels. Twenty, 30, or $40 \mu \mathrm{M}$ of tamoxifen treatment also increased the amounts of cleaved PARP and caspase- 8 in the MCF-7 cells, but decreased their expression of $\beta$-catenin. The protein expression of E-cadherin was decreased in MCF-7 cells after treatment with 30 or $40 \mu \mathrm{M}$ of tamoxifen. We also examined whether the longer exposure to tamoxifen affects the expression of claudin-1 in MCF-7 cells. The expression of claudin- 1 and the amount of cleaved PARP were significantly increased in the cells treated with $20 \mu \mathrm{M}$ of tamoxifen for $48 \mathrm{~h}$ (Figure 1C and 1D). In T47 D cells, the amounts of cleaved PARP and caspase- 8 were increased, whereas the expression of E-cadherin was decreased after $40 \mu \mathrm{M}$ of tamoxifen treatment. However, the protein expression levels of claudin-1, claudin-4, claudin-7, and $\beta$-catenin in T47 D cells were almost unaffected by tamoxifen treatment. We also examined whether the expression of claudin-1 mRNA was affected by tamoxifen treatment. MCF-7 and T47 D cells were treated with various concentrations of tamoxifen for $20 \mathrm{~h}$, and RNA samples were prepared from the cells and subjected to RT-PCR and real-time PCR analyses for claudin-1, claudin-4, E-cadherin, glyceraldehyde-3phosphate dehydrogenase (GAPDH), and $18 \mathrm{~S}$ rRNA (Figure 2A and 2B). The mRNA levels of claudin-1 were significantly increased in MCF-7 cells after treatment with 30 or $40 \mu \mathrm{M}$ of tamoxifen, whereas tamoxifen treatment did not affect the expression of claudin-1 in T47 D cells. The mRNA expression of E-cadherin was decreased in both MCF-7 and T47 D cells after treatment with $40 \mu \mathrm{M}$ of tamoxifen. On the other hand, tamoxifen treatment did not affect the expression of claudin-4 in MCF-7 or T47 D cells.

Nuclear condensation is one of the features of apoptosis. Using immunofluorescent staining, we examined whether tamoxifen treatment induces nuclear condensation. MCF-7 and T47 D cells were treated with tamoxifen, fixed, and then stained with Hoechst 33258. Nuclear condensation was increased about 9 or 5 -fold in MCF-7 or T47 D cells, respectively, treated with $40 \mu \mathrm{M}$ of tamoxifen compared with that in the untreated-control cells (Figure 3A and 3B).

\section{Claudin-1 has anti-apoptotic effects under tamoxifen treatment in MCF-7 cells}

To understand the mechanism of the upregulation of claudin-1 expression by tamoxifen treatment, we examined whether the transfection of claudin-1 siRNA affected the expression of factors related to apoptosis. MCF-7 cells were transfected with control siRNA or siRNA against claudin-1. After $48 \mathrm{~h}$ of transfection, the cells were treated with $40 \mu \mathrm{M}$ of tamoxifen for $20 \mathrm{~h}$. The cell lysates were then subjected to Western blot analyses for claudin-1, cleaved PARP, cleaved caspase-8, 
A
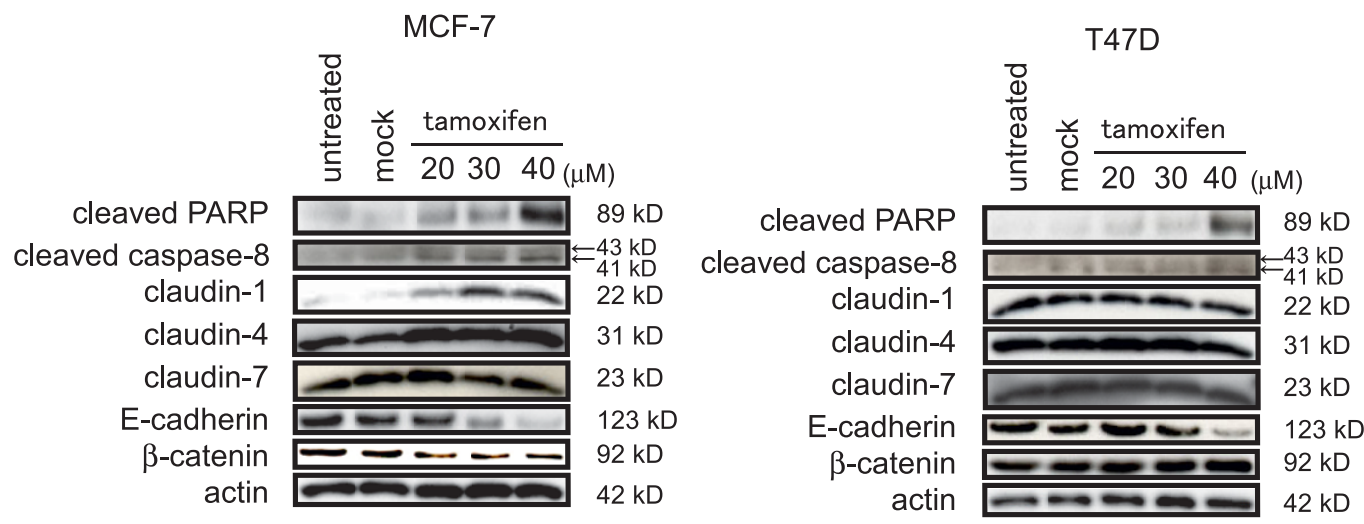

B

MCF-7
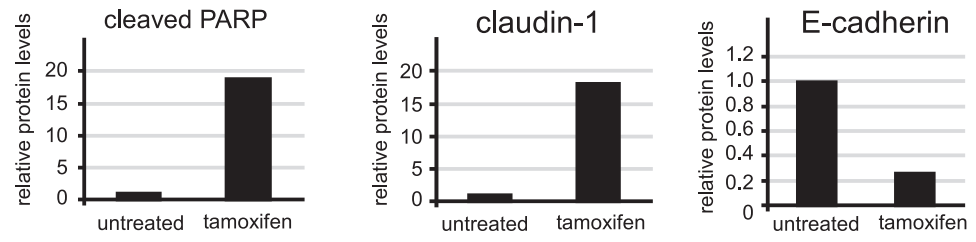

T47D
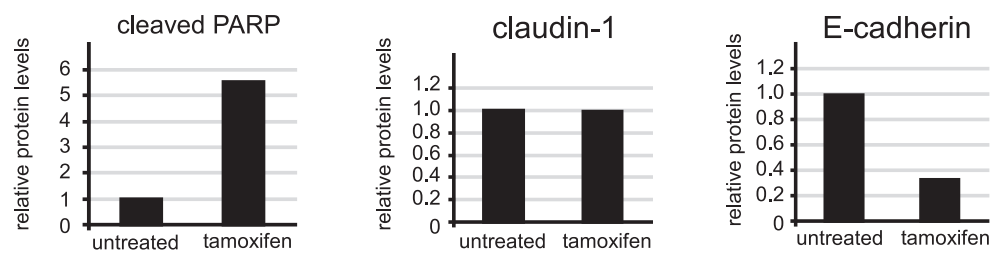

C
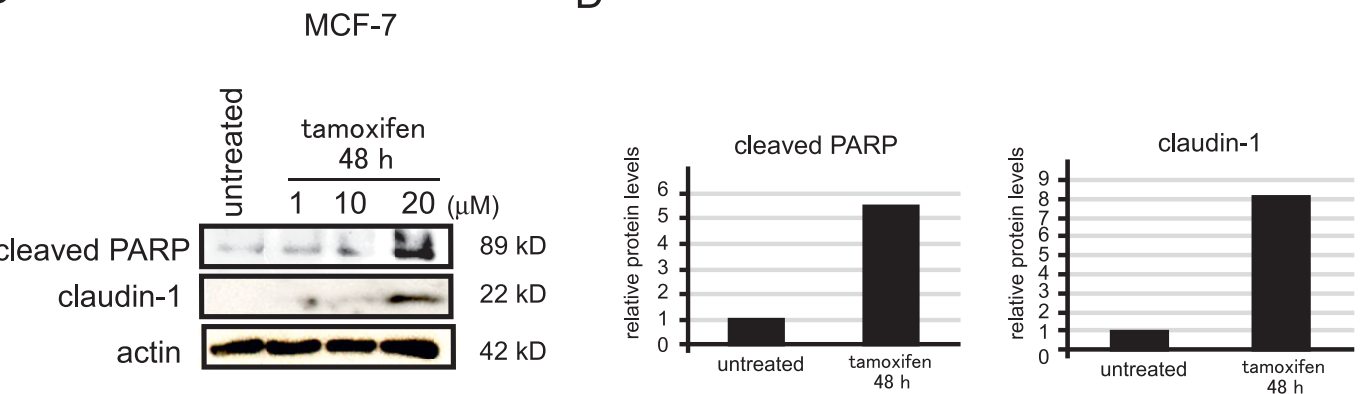

Figure 1 The protein levels of claudin-1 were increased in MCF-7 cells treated with tamoxifen. (A) MCF-7 and T47 D cells were treated with the control treatment (buffer alone: Mock) for $20 \mathrm{~h}$ or 20, 30, or $40 \mu \mathrm{M}$ of tamoxifen for $20 \mathrm{~h}$, cell lysates were prepared and subjected to Western blot analyses for cleaved PARP, claudin-1, claudin-4, claudin-7, E-cadherin, cleaved caspase-8, and actin. One representative of at least three independent experiments with similar results is shown. (B) The intensity of the bands for cleaved PARP, claudin-1 and E-cadherin in untreated and tamoxifen $(40 \mu \mathrm{M})$ - treated cells were quantified. (C) MCF-7 cells were treated with or without 1, 10 or $20 \mu \mathrm{M}$ of tamoxifen for $48 \mathrm{~h}$, cell lysates were prepared and subjected to Western blot analyses for cleaved PARP, claudin-1 and actin. One representative of at least three independent experiments with similar results is shown. (D) The intensity of the bands for cleaved PARP and claudin-1 in untreated and tamoxifen $(40 \mu \mathrm{M})$ - treated cells were quantified. 


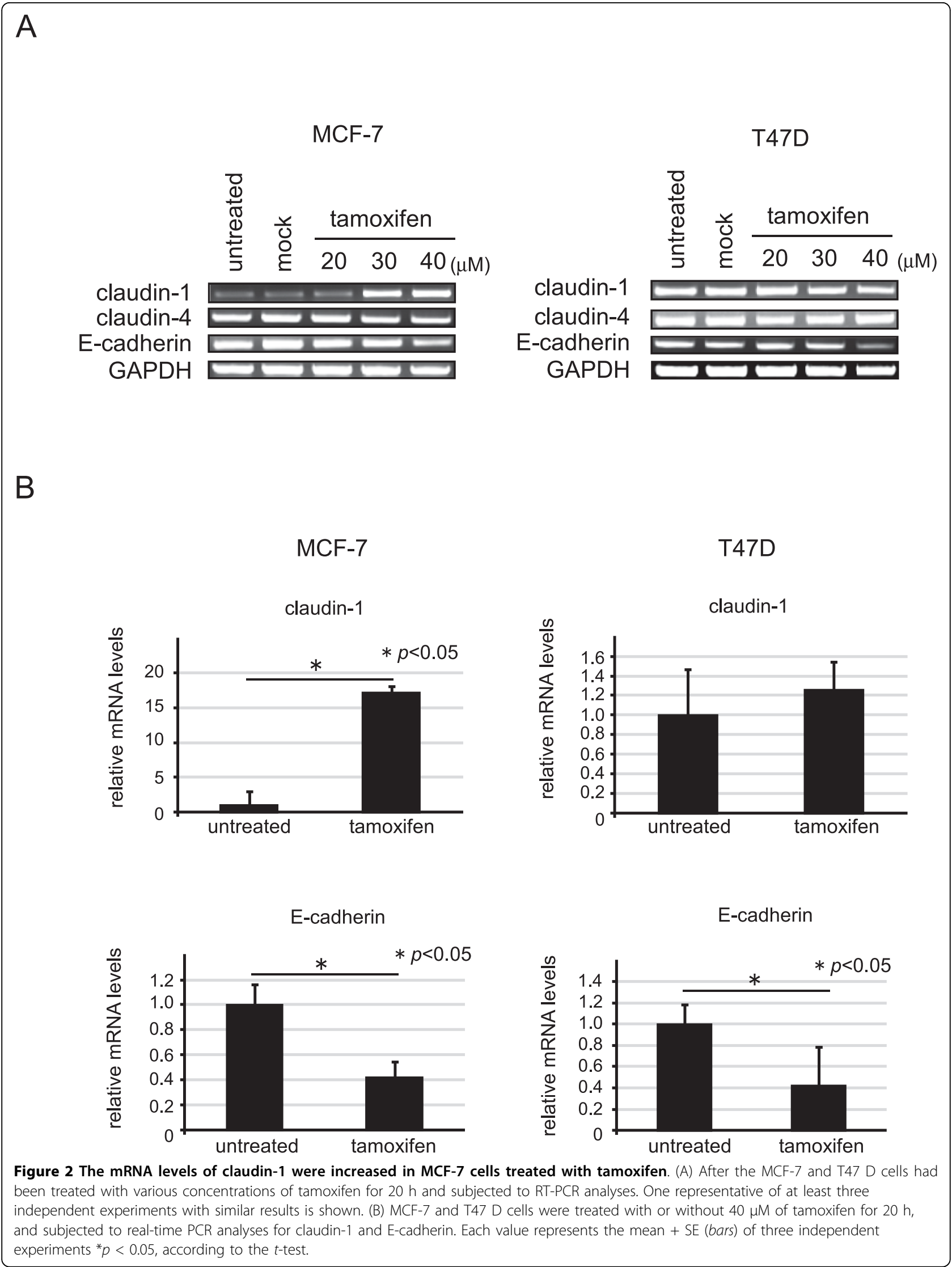


A

MCF-7

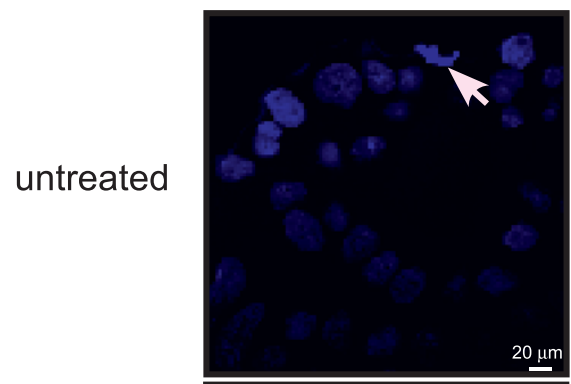

tamoxifen

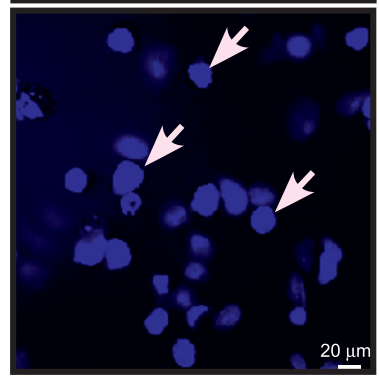

T47D
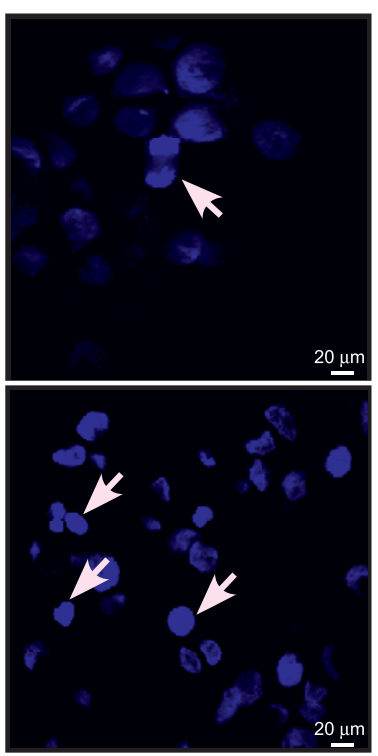

B

MCF-7

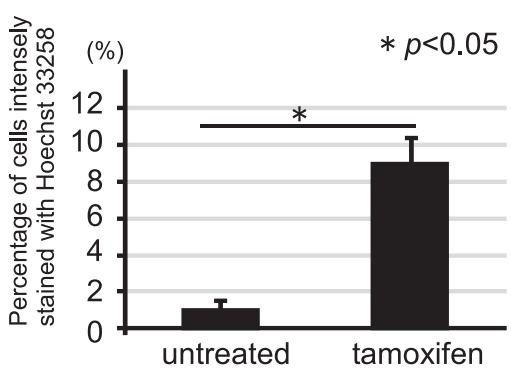

T47D

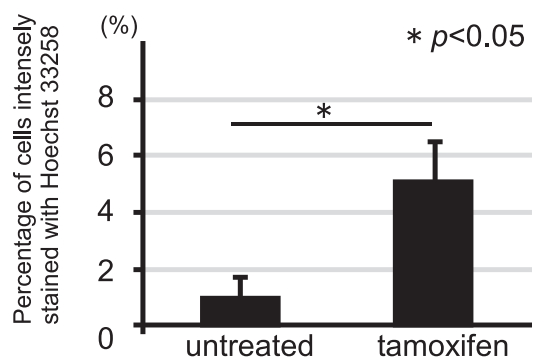

Figure 3 Nuclear condensation was detected by tamoxifen treatment both in MCF-7 and T47 D cells. (A) MCF-7 and T47 D cells were seeded in a 4-chamber slide glass and incubated overnight. The cells were treated with or without $40 \mu \mathrm{M}$ of tamoxifen for $20 \mathrm{~h}$, fixed, and stained with Hoechst 33258. The arrows show nuclear condensation. (B) Percentage of cells intensely fragmented and condensed in nuclei with Hoechst 33258 were counted as positive cells. About 100 total cells were counted in individual ten random microscopic fields at $\times 40$ magnification. Each value represents the mean $+\mathrm{SE}$ (bars) of two independent experiments ${ }^{*} p<0.05$, according to the $t$-test.

E-cadherin, $\beta$-catenin, Bax, Bcl-2, cyclinD1, p53, p21, and actin (Figure 4A and 4B). Claudin-1 knockdown by siRNA significantly reduced the expression of claudin-1 with or without tamoxifen treatment, and the transfection of claudin-1 siRNA increased the amounts of cleaved PARP and caspase- 8 with or without tamoxifen treatment. The expression of E-cadherin was upregulated by claudin-1 knockdown without tamoxifen treatment, while the expression of E-cadherin in the presence of claudin-1 siRNA and tamoxifen treatment was slightly increased. On the other hand, the expression of cyclinD1 was downregulated by claudin-1 
A

\section{MCF-7}
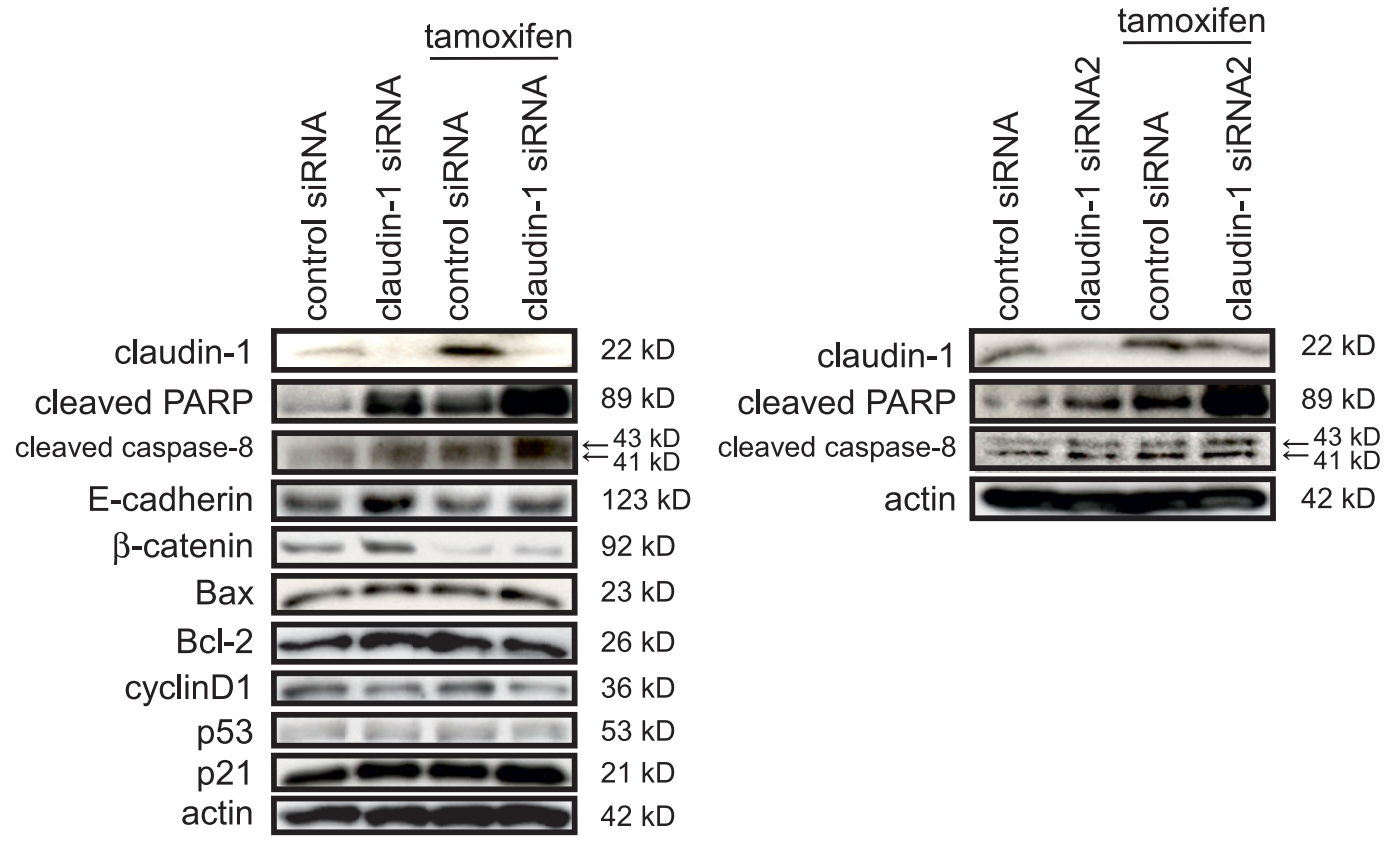

B

MCF-7
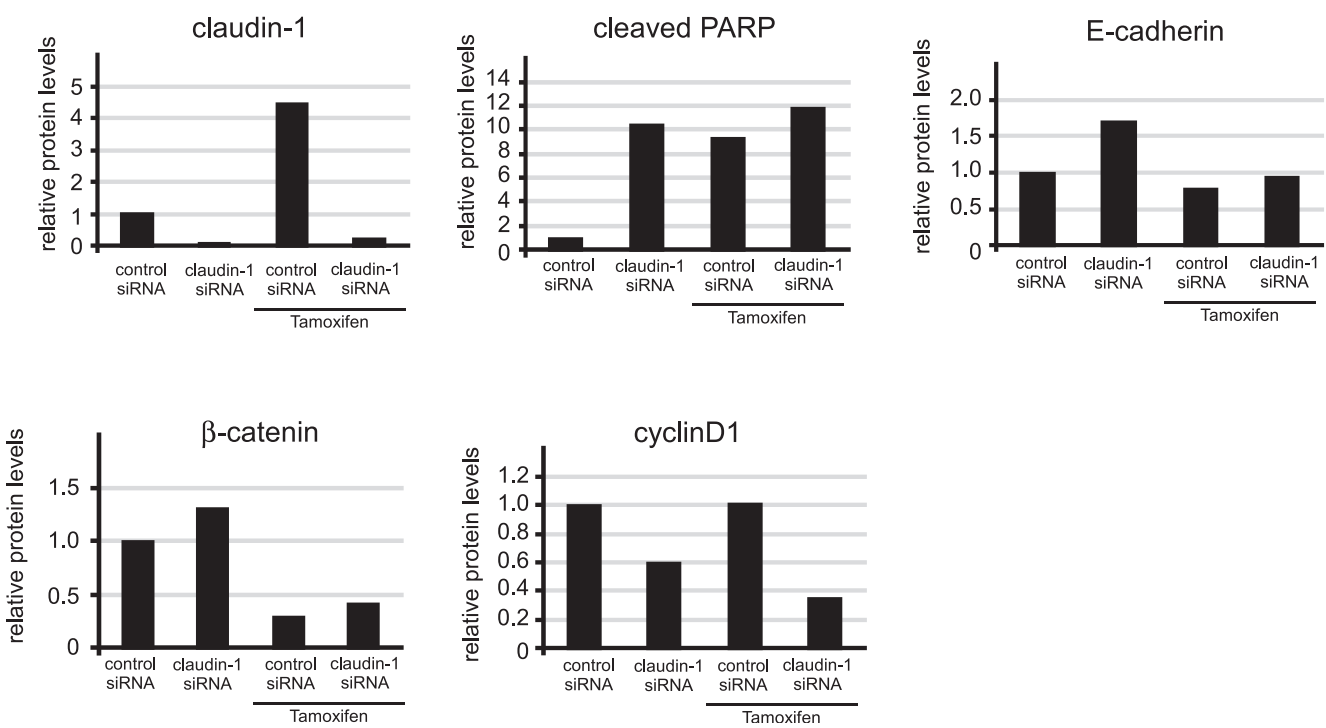

Figure 4 Anti-apoptotic effect of claudin-1 induced by tamoxifen in MCF-7 cells. (A) MCF-7 cells were transfected with control siRNA or siRNAs against claudin- 1 (left: claudin-1 siRNA, right: claudin-1 siRNA2) and incubated for $48 \mathrm{~h}$, before being treated with or without $40 \mu \mathrm{M}$ of tamoxifen and then incubated for a further $20 \mathrm{~h}$. Cell lysates were prepared from the cells and subjected to Western blot analyses for claudin-1, cleaved PARP, cleaved caspase-8, E-cadherin, $\beta$-cadherin, Bax, Bcl-2, cyclinD1, p53, p21, and actin. One representative of at least three independent experiments with similar results is shown. (B) The intensity of the bands for claudin-1, cleaved PARP, E-cadherin, $\beta$-cadherin and cyclinD1 in control siRNA and claudin-1 siRNA - treated cells with or without tamoxifen $(40 \mu \mathrm{M})$ for $20 \mathrm{~h}$ were quantified. 


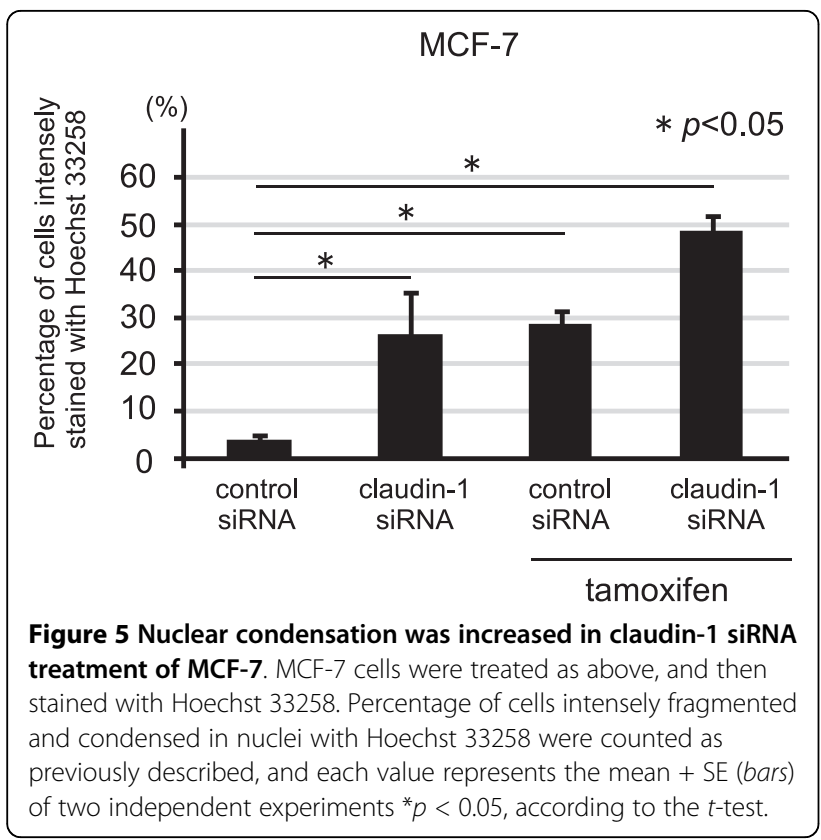

knockdown with or without tamoxifen treatment, whereas tamoxifen treatment did not affect the expression of cyclinD1. The expression of $\beta$-catenin was upregulated by claudin-1 knockdown in the absence of tamoxifen treatment, but combination treatment involving claudin-1 siRNA and tamoxifen had little effect the expression of $\beta$-catenin. The expression levels of Bax, Bcl-2, p53, p21, and actin were not affected by claudin-1 knockdown, tamoxifen treatment or combination treatment involving claudin-1 siRNA and tamoxifen.

As nuclear condensation was induced in the cells treated with tamoxifen (Figure 3), we examined whether claudin-1 siRNA treatment had similar effects. Nuclear condensation was increased about 7 -fold in the MCF-7 cells treated with claudin-1 siRNA as well as the control siRNA and tamoxifen-treated cells compared with that in the control (only control siRNA treatment) cells (Figure 5). Combination treatment involving claudin-1 siRNA and tamoxifen increased nuclear condensation about 16-fold compared with that in the control cells.

Next, we examined whether claudin-1 knockdown by siRNA affected the amount of cleaved PARP in T47 D cells. The amount of cleaved PARP was upregulated by tamoxifen treatment, but it was little affected by claudin1 siRNA treatment or combination treatment involving claudin-1 siRNA and tamoxifen (Figure 6A and 6B).

\section{Changes in the subcellular localization of E-cadherin and $\beta$-catenin in MCF-7 cells treated with claudin-1 siRNA or tamoxifen}

We investigated whether the subcellular localization of E-cadherin and $\beta$-catenin was affected by claudin- 1
siRNA or tamoxifen treatment using immunofluorescent staining. As shown in Figure 7A and 8A, tamoxifen treatment increased the amount of claudin-1 in the cell membranes of MCF-7 cells, while tamoxifen treatment did not affect the amount of claudin-1 in T47 D cells. Tamoxifen treatment decreased the amounts of E-cadherin in the cell membranes of MCF-7 and T47 D cells, while it increased the amount of E-cadherin in the cytoplasm of MCF-7, but not T47 D, cells. On the other hand, tamoxifen treatment decreased the amount of $\beta$-catenin in the cell membranes of MCF-7 cells, but increased it in the cytoplasm. The amount of E-cadherin in the cell membrane was decreased in MCF-7 cells treated with claudin-1 siRNA, while the amount of E-cadherin in the cytoplasm was increased (Figure 7B). Knockdown of claudin-1 also increased the amount of $\beta$-catenin in the cell membranes of MCF-7 cells. In T47 D cells, claudin-1 siRNA treatment did not affect the amount of E-cadherin or $\beta$-catenin in the cell membrane or cytoplasm (Figure $8 \mathrm{~B}$ ). These data demonstrated that claudin-1 has anti-apoptotic effects during tamoxifen treatment, which involve changes in the subcellular localization of claudin-1, E-cadherin, and $\beta$-catenin in MCF-7 cells.

\section{Discussion}

In this study, we focused on the functions of claudin-1 in human breast cancer cells. Claudins are generally located in the cell membrane and mainly contribute to cell-cell adhesion $[18,19]$. It was confirmed that claudin1 is localized to the cell membrane in T47 D cells. However, little claudin-1 was localized to the cell membrane in MCF-7 cells. Tamoxifen treatment increased claudin1 protein expression as well as its membrane localization in MCF-7 cells, whereas tamoxifen treatment did not affect the expression or subcellular localization of claudin-1 in T47 D cells. Thus, the function of claudin1 may differ among different cell types. It has been reported that MCF-7 cells have wild type p53 but lack caspase- 3 . On the other hand, T47 D cells express caspase- 3 but p53 is mutated [20,21]. They showed that the sensitivity of these cells against anti-cancer drugs such as staurosporine and Triphala are different. The differential expression of claudin-1 may be also related to differences in phenotype of these two cell lines.

Recent studies have shown the relationship between claudin expression and cellular resistance in tumors $[22,23]$. The elevated claudin-1 expression induced by 5 -fluorouracil (5-FU) or TNF- $\alpha$ treatment is associated with the regulation of apoptosis in nasopharyngeal carcinoma and pancreatic cancer cells, although these cells low levels of protein expression and claudin-1 localization in the membrane were also observed $[23,24]$. In addition, knockdown of claudin- 6 induces cellular 


\section{A}

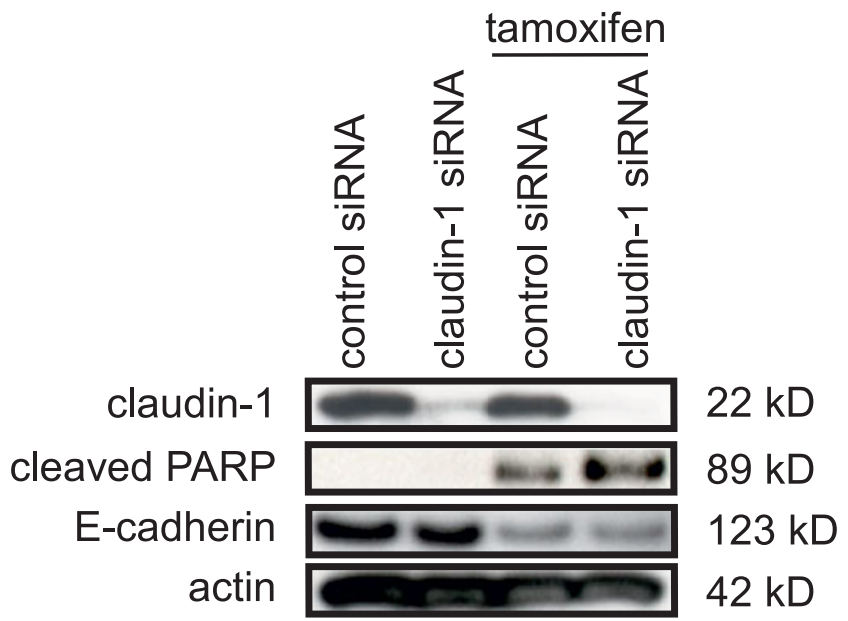

B

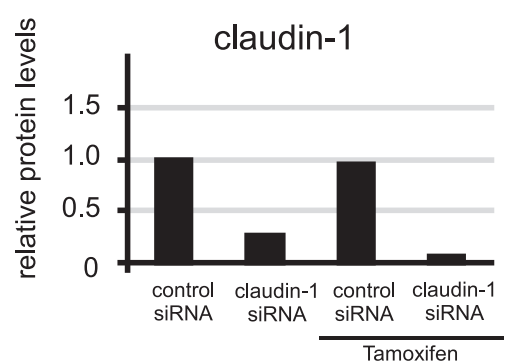

T47D

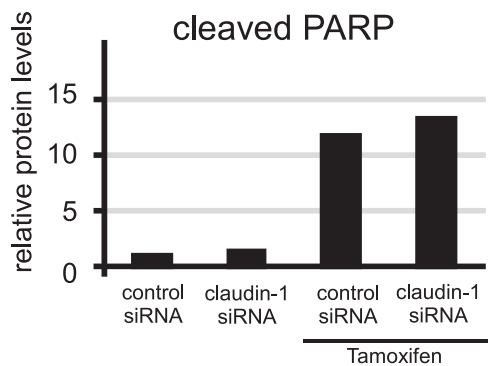

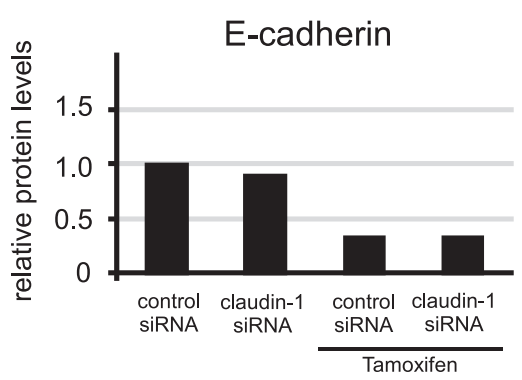

Figure 6 Claudin-1 knockdown has little effects on apoptosis in T47 D cells. (A) T47 D cells were treated with control siRNA or siRNA against claudin-1 and treated as above. Cell lysates were subjected to Western blot analyses for claudin-1, cleaved PARP, E-cadherin, and actin. One representative of at least three independent experiments with similar results is shown. (B) The intensity of the bands for claudin-1, cleaved PARP and E-cadherin in control siRNA and claudin-1 siRNA - treated cells with or without tamoxifen $(40 \mu \mathrm{M})$ for $20 \mathrm{~h}$ were quantified.

resistance to apoptosis in MCF-7 cells [22]. These observations and our findings suggest that the upregulation of claudin-1 by apoptosis-inducers contributes to cellular resistance to apoptosis when claudin-1 protein is expressed at low levels and mislocalized to the cell membrane. However, it is unclear how claudin-1 is regulated by apoptosis inducers. We found that tamoxifen treatment increased the expression of claudin-1 mRNA and proteins related to apoptosis in MCF-7 cells.
We speculate that tamoxifen treatment regulates the transcription of claudin-1. Further studies are needed to interpret whether tamoxifen treatment regulates claudin-1 expression.

Next, we investigated the molecular mechanisms of the apoptosis induced by claudin-1 knockdown in MCF7 cells. Unfortunately, apoptosis-related proteins, such as Bax, Bcl-2, p53, and p21, were not affected by claudin-1 knockdown with or without tamoxifen treatment. 
A

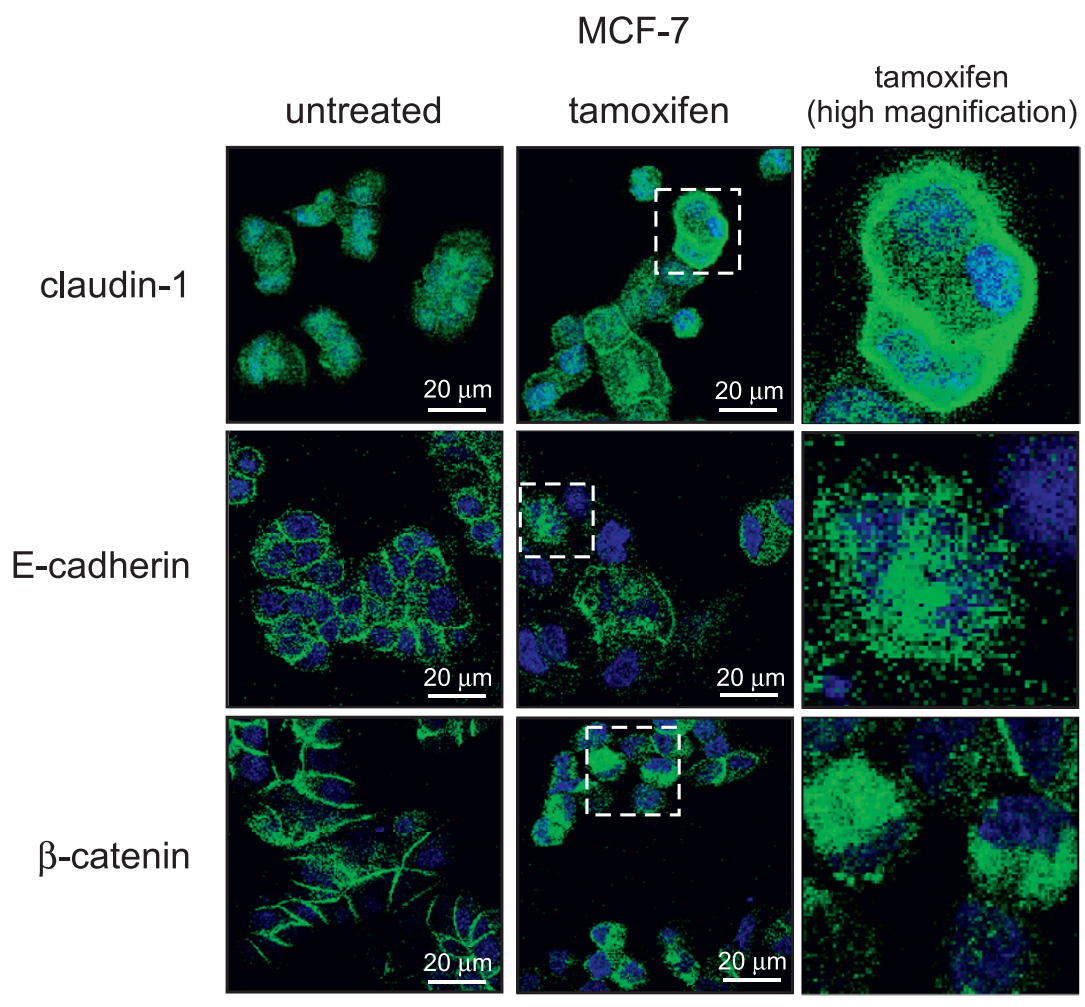

B

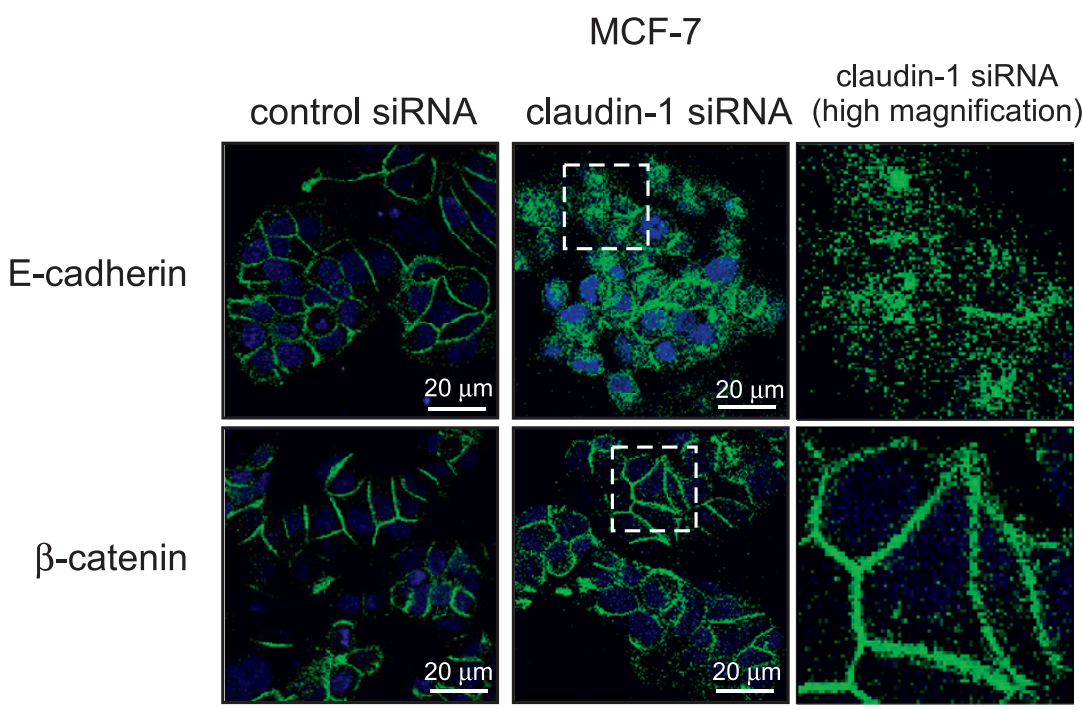

Figure 7 Subcellular localization of claudin-1, E-cadherin, and $\beta$-catenin in MCF-7 cells after tamoxifen or claudin-1 siRNA treatment. (A) MCF-7 cells were treated with or without $40 \mu \mathrm{M}$ of tamoxifen for $20 \mathrm{~h}$, fixed, incubated with anti-claudin-1, E-cadherin or $\beta$-catenin antibody, and visualized using Alexa488-conjugated secondary antibody (Green). The cells were also counterstained with DAPI (Blue) in order to localize the nucleus. A merged image that is representative of at least two independent experiments with similar results is shown. White dot box for high magnification were also shown. (B) MCF-7 cells were transfected with siRNA against claudin-1. After $48 \mathrm{~h}$ incubation, the cells were fixed, incubated with anti-E-cadherin or $\beta$-catenin antibody, and visualized using Alexa488. One representative merged image is shown. 
A

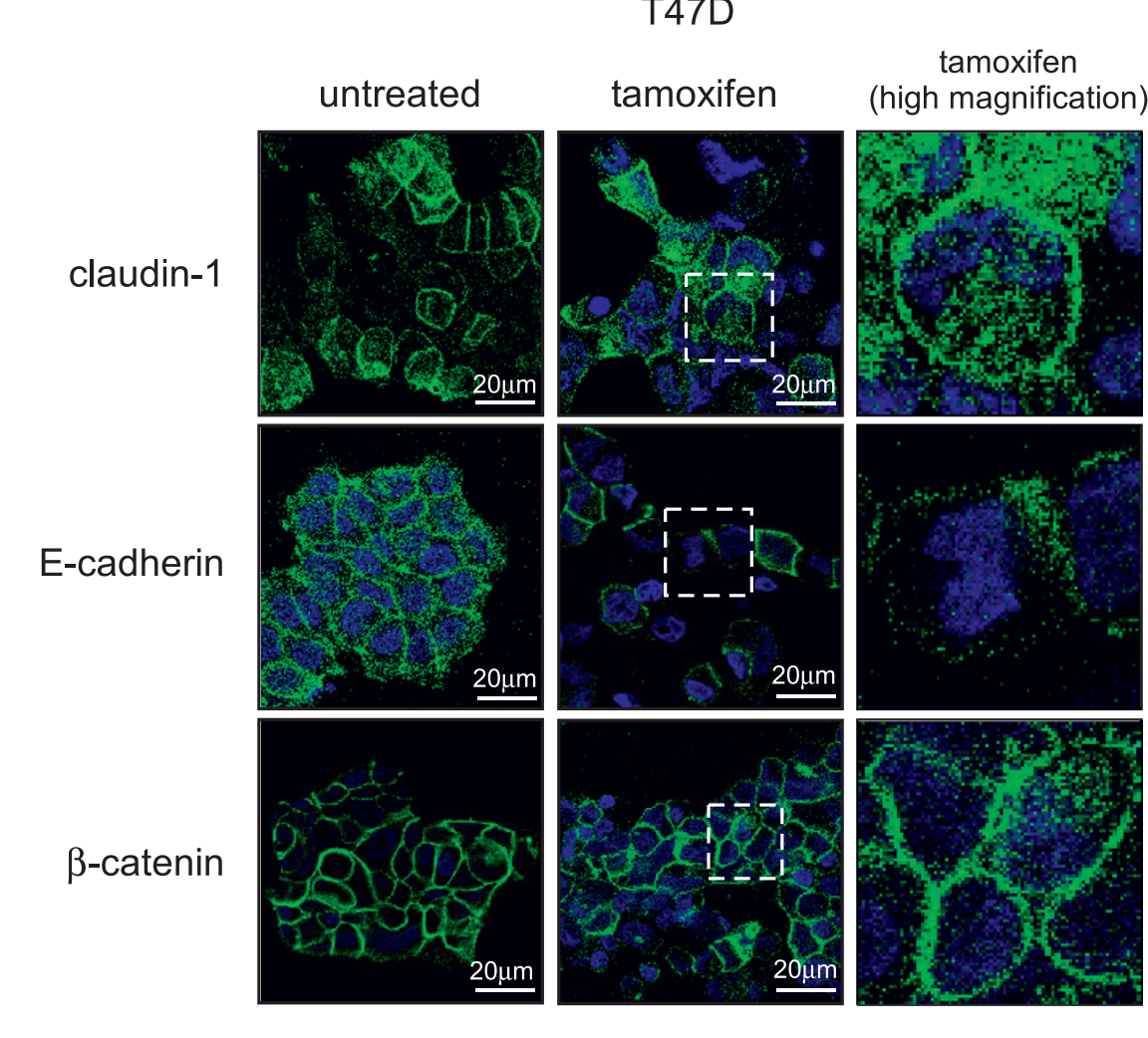

B

\section{T47D}

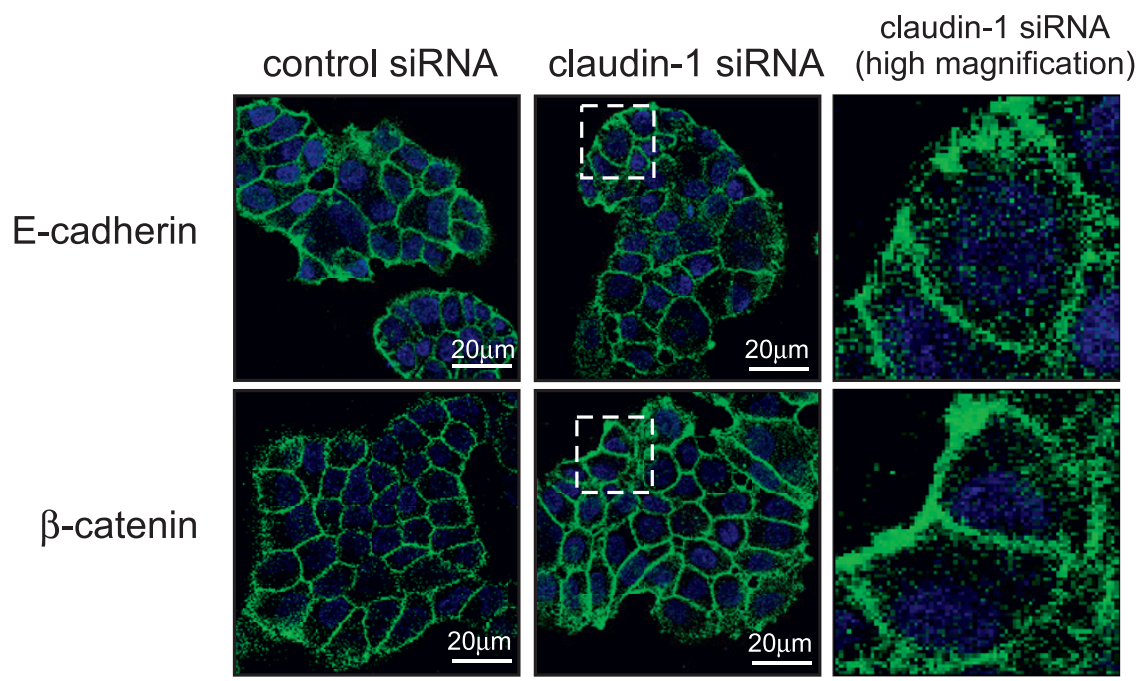

Figure 8 Subcellular localization of claudin-1, E-cadherin, and $\beta$-catenin in T47 D cells after tamoxifen or claudin-1 siRNA treatment (A) T47 D cells were treated with or without $40 \mu \mathrm{M}$ of tamoxifen for $20 \mathrm{~h}$, incubated with anti-claudin-1, E-cadherin, or $\beta$-catenin antibody, and visualized using Alexa488. One representative merged image is shown. (B) T47 D cells were transfected with siRNA against claudin-1. After $48 \mathrm{~h}$ incubation, the cells were fixed, incubated with anti-E-cadherin or $\beta$-catenin antibody, and visualized using Alexa488. One representative merged image is shown. 
However, the expression of cyclin D1 was downregulated by claudin-1 knockdown, regardless of tamoxifen treatment in MCF-7 cells. We speculate that the regulation of apoptosis by claudin-1 knockdown may be related to pathways other than the $\mathrm{p} 21, \mathrm{p} 53$, and mitochondrial-pathways. Lee et al. showed that claudin-1 has anti-apoptotic effects under 5-FU treatment, but they could not demonstrate the molecular mechanisms of claudin-1 induced apoptosis [23].

Interestingly, it has been reported that changes in the subcellular localization of $\beta$-catenin or E-cadherin may be related to the regulation of apoptosis [25-28]. 2 -methoxyestradiol induces $\beta$-catenin expression in prostate cancer cells, but blocks $\beta$-catenin degradation, as well as its cytoplasmic or nuclear accumulation, resulting in cell cycle arrest and apoptosis [29]. Therefore, we performed immunofluorescent staining to analyze the changes in the subcellular localization of $\beta$-catenin and E-cadherin induced by claudin-1 knockdown or tamoxifen treatment. As expected, claudin-1 knockdown affected the subcellular localization of $\beta$-catenin and E-cadherin in MCF-7, but not T47 D cells. Tamoxifen treatment also affected the subcellular localization of $\beta$-catenin and E-cadherin. So, we speculate that knockdown of claudin-1 upregulates the protein expression of $\beta$-catenin and changes its subcellular localization in MCF-7 cells and then induces cell cycle arrest, resulting in apoptosis. However, tamoxifen treatment downregulates the expression of $\beta$-catenin in MCF-7 cells. According to these results, we suggest that tamoxifen treatment upregulates the expression of claudin- 1 and that the upregulation of claudin-1 subsequently downregulates the expression of $\beta$-catenin. $\beta$-catenin may be one of the downstream factors of claudin-1 in MCF-7 cells. However, the detailed mechanism by which claudin-1 regulates the expression of $\beta$-catenin needs to be clarified.

We also examined whether other claudins are affected by tamoxifen treatment. The expression of claudin- 4 and claudin-7 was not affected by tamoxifen treatment in MCF-7 and T47 D cells as shown in Figure 1A and $2 \mathrm{~A}$. Thus, only claudin- 1 in claudin's family would be specifically affected by tamoxifen treatment, although we could not elucidate the specific effect of claudin-1 by tamoxifen treatment.

In the present study, we demonstrated the function of claudin-1 in human breast cancer MCF-7 cells. Claudin1 has anti-apoptotic effects in tamoxifen-treated MCF-7 cells.

\section{Conclusion}

We demonstrated the function of claudin-1 in human breast cancer MCF-7 cells. Our results showed for the first time that claudin-1 has anti-apoptotic effects in tamoxifen-treated MCF-7 cells, involving the regulation of apoptosis-related factors and subcellular localization of adherens junctions.

Our data would be useful for future studies in order to establish the mechanisms of apoptosis regulation in human breast cancer. Further research needs to be clarified the relationship between tight junctions and apoptosis.

\section{Abbreviations}

RT-PCR: Reverse transcription-polymerase chain reaction; PARP: poly (ADPribose) polymerase; 5-FU: 5-fluorouracil; TNF- $\alpha$ : tumor necrosis factor-alpha; PBS: phosphate-buffered saline

\section{Acknowledgements}

This study was supported by Grants-in-Aid for Science from the Ministry of Education, Culture, Sports, Science, and Technology of Japan; a Grant for the Hirosaki University Institutional Research; the Fund for the Promotion of International Scientific Research; and the Karoji Memorial Fund for Medical Research.

\section{Author details}

${ }^{1}$ Department of Pathology and Bioscience, Hirosaki University Graduate School of Medicine, Hirosaki 036-8562, Japan. Department of Pathology, College of Basic Medical Sciences, China Medical University, Shenyang, 110001, China. ${ }^{3}$ Department of Surgery, Hirosaki University Graduate School of Medicine, Hirosaki 036-8562, Japan.

\section{Authors' contributions}

HA performed major experimental work. FS designed HA's original work and helped HA's work and completed manuscript. KH and HK corrected HA's manuscript. SM, YW, YL, JK and HO helped HA's work. All authors read and approved the final manuscript.

\section{Competing interests}

The authors declare that they have no competing interests.

Received: 8 May 2010 Accepted: 12 October 2010

Published: 12 October 2010

\section{References}

1. Jemal A, Siegel R, Ward E, Hao Y, Xu J, Thun MJ: Cancer statistics, 2009. CA Cancer J Clin 2009, 59:225-249.

2. Sjöström J, Bergh J: How apoptosis is regulated, and what goes wrong in cancer. BMJ 2001, 322:1538-1539, Review.

3. Chang J, Powles TJ, Allred DC, Ashley SE, Makris A, Gregory RK, Osborne CK, Dowsett M: Prediction of clinical outcome from primary tamoxifen by expression of biologic markers in breast cancer patients. Clin Cancer Res 2000, 6:616-621.

4. Sutherland RL, Green MD, Hall RE, Reddel RR, Taylor IW: Tamoxifen induces accumulation of MCF 7 human mammary carcinoma cells in the G0/G1 phase of the cell cycle. Eur J Cancer Clin Oncol 1983, 19:615-621.

5. Perry RR, Kang Y, Greaves B: Effects of tamoxifen on growth and apoptosis of estrogen-dependent and -independent human breast cancer cells. Ann Surg Oncol 1995, 2:238-245.

6. Martin G, Melito G, Rivera E, Levin E, Davio C, Cricco G, Andrade N, Caro R, Bergoc R: Effect of tamoxifen on intraperitoneal N-nitroso-N-methylurea induced tumors. Cancer Lett 1996, 100:227-234.

7. Lagadec C, Adriaenssens E, Toillon RA, Chopin V, Romon R, Van Coppenolle F, Hondermarck H, Le Bourhis X: Tamoxifen and TRAIL synergistically induce apoptosis in breast cancer cells. Oncogene 2008 27:1472-1477.

8. Kang Y, Cortina R, Perry RR: Role of c-myc in tamoxifen-induced apoptosis estrogen-independent breast cancer cells. J Natl Cancer Inst 1996 88:224-226.

9. Duh JL, Yu R, Jiao JJ, Matwyshyn GA, Li W, Tan TH, Kong AN: Activation of signal transduction kinases by tamoxifen. Pharm Res 1997, 14:186-189. 
10. Mandlekar S, Yu R, Tan TH, Kong AN: Activation of caspase-3 and c-Jun $\mathrm{NH} 2$-terminal kinase -1 signaling pathways in tamoxifen-induced apoptosis of human breast cancer cells. Cancer Res 2000, 60:5995-6000.

11. Ichikawa A, Ando J, Suda K: G1 arrest and expression of cyclin-dependent kinase inhibitors in tamoxifen-treated MCF-7 human breast cancer cells. Hum Cell 2008, 21:28-37.

12. Cereijido M, Contreras RG, Shoshani L, Flores-Benitez D, Larre I: Tight junction and polarity interaction in the transporting epithelial phenotype. Biochim Biophys Acta 2008, 1778:770-793, Review.

13. Dhawan $P$, Singh AB, Deane NG, No Y, Shiou SR, Schmidt C, Neff J, Washington MK, Beauchamp RD: Claudin-1 regulates cellular transformation and metastatic behavior in colon cancer. J Clin Invest 2005, 115:1765-1776.

14. Usami Y, Chiba H, Nakayama F, Ueda J, Matsuda Y, Sawada N, Komori T, Ito A, Yokozaki H: Reduced expression of claudin-7 correlates with invasion and metastasis in squamous cell carcinoma of the esophagus. Hum Pathol 2006, 37:569-577.

15. Oliveira SS, Morgado-Díaz JA: Claudins: multifunctional players in epithelial tight junctions and their role in cancer. Cell Mol Life Sci 2007, 64:17-28.

16. Tokés AM, Kulka J, Paku S, Szik A, Páska C, Novák PK, Szilák L, Kiss A, Bögi K, Schaff Z: Claudin-1, -3 and -4 proteins and mRNA expression in benign and malignant breast lesions: a research study. Breast Cancer Res 2005, 7: R296-R305.

17. Morohashi S, Kusumi T, Sato F, Odagiri H, Chiba H, Yoshihara S, Hakamada K, Sasaki M, Kijima H: Decreased expression of claudin-1 correlates with recurrence status in breast cancer. Int J Mol Med 2007 20:139-143.

18. Furuse $M$, Sasaki $H$, Fujimoto $K$, Tsukita S: A single gene product, claudin-1 or -2 , reconstitutes tight junction strands and recruits occludin in fibroblasts. J Cell Biol 1998, 143:391-401.

19. Tsukita S, Furuse M: Overcoming barriers in the study of tight junction functions: from occludin to claudin. Genes Cells 1998, 3:569-573, Review.

20. Mooney LM, Al-Sakkaf KA, Brown BL, Dobson PR: Apoptotic mechanisms in T47 D and MCF-7 human breast cancer cells. Br J Cancer 2002, 87:909-917.

21. Sandhya T, Mishra KP: Cytotoxic response of breast cancer cell lines, MCF 7 and T $47 \mathrm{D}$ to triphala and its modification by antioxidants. Cancer letter 2006, 238:304-313.

22. Osanai M, Murata M, Chiba H, Kojima T, Sawada N: Epigenetic silencing of claudin- 6 promotes anchorage-independent growth of breast carcinoma cells. Cancer Sci 2007, 98:1557-1562.

23. Lee JW, Hsiao WT, Chen HY, Hsu LP, Chen PR, Lin MD, Chiu SJ, Shih WL, Hsu YC: Upregulated claudin-1 expression confers resistance to cell death of nasopharyngeal carcinoma cells. Int J Cancer 2010, 126:1353-1366

24. Kondo J, Sato F, Kusumi T, Liu Y, Motonari O, Sato T, Kijima H: Claudin-1 expression is induced by tumor necrosis factor-alpha in human pancreatic cancer cells. Int J Mol Med 2008, 22:645-649.

25. Yang SZ, Kohno N, Kondo K, Yokoyama A, Hamada H, Hiwada K, Miyake M: Adriamycin activates E-cadherin-mediated cell-cell adhesion in human breast cancer cells. Int J Oncol 1999, 15:1109-1115.

26. Peluso JJ, Pappalardo A, Hess SA: Effect of disrupting cell contact on the nuclear accumulation of beta-catenin and subsequent apoptosis of rat ovarian surface epithelial cells in vitro. Endocrine 2000, 12:295-302.

27. Wang D, Xiang DB, He YJ, Li ZP, Wu XH, Mou JH, Xiao HL, Zhang QH: Effect of caffeic acid phenethyl ester on proliferation and apoptosis of colorectal cancer cells in vitro. World J Gastroenterol 2005, 11:4008-4012.

28. He YJ, Liu BH, Xiang DB, Qiao ZY, Fu T, He YH: Inhibitory effect of caffeic acid phenethyl ester on the growth of SW480 colorectal tumor cells involves beta-catenin associated signaling pathway down-regulation. World J Gastroenterol 2006, 12:4981-4985.

29. Van Veldhuizen PJ, Ray G, Banerjee S, Dhar G, Kambhampati S, Dhar A Banerjee SK: 2-Methoxyestradiol modulates beta-catenin in prostate cancer cells: a possible mediator of 2-methoxyestradiol-induced inhibition of cell growth. Int J Cancer 2008, 122:567-571.

\section{Pre-publication history}

The pre-publication history for this paper can be accessed here: http://www.biomedcentral.com/1471-2407/10/548/prepub

\section{doi:10.1186/1471-2407-10-548}

Cite this article as: Akasaka et al:: Anti-apoptotic effect of claudin-1 in tamoxifen-treated human breast cancer MCF-7 cells. BMC Cancer 2010 10:548.

\section{Submit your next manuscript to BioMed Central and take full advantage of:}

- Convenient online submission

- Thorough peer review

- No space constraints or color figure charges

- Immediate publication on acceptance

- Inclusion in PubMed, CAS, Scopus and Google Scholar

- Research which is freely available for redistribution 